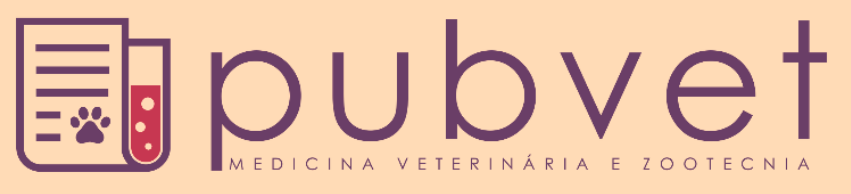

https://doi.org/10.31533/pubvet.v14n12a714.1-5

\title{
Postoplastia em touro Nelore acometido com acrobustite: Relato de caso
}

\author{
José Pires de Carvalho Neto ${ }^{1 *}$, Pedro Ferreira de Sousa Junior ${ }^{2} \bullet$, Leontina Nascimento \\ Ribeiro $^{2}{ }^{\bullet}$, Jackson Breno Gomes Dantas ${ }^{2}$, Manoel Lopes da Silva Filho ${ }^{\circ}{ }^{\circ}$, Sued Felipe Lacerda

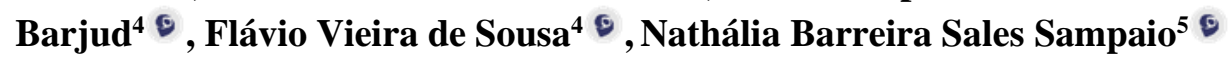

\author{
${ }^{I}$ Doutorando em Ciências Animal da Universidade Federal do Piauí UFPI, Campus Ministro Petrônio Portella \\ ${ }^{2}$ Discente do curso de Medicina Veterinária da Universidade Federal do Piauí, Bom Jesus-PI. Brasil. \\ ${ }^{3}$ Docente Titular da Universidade Federal do Piauí, Bom Jesus-PI. Brasil. \\ ${ }^{4}$ Mestre em Zootecnia da Universidade Federal do Piauí UFPI/CPCE. \\ ${ }^{5}$ Graduada em Medicina Veterinária da Universidade Federal do Piauí UFPI/CPCE. \\ *Autor para correspondência.E-mail: josepires@ufpi.edu.br
}

Resumo. Objetivou-se com esse trabalho descrever um caso de acrobustite, caracterizada pela inflamação da extremidade do prepúcio em touros, causando infertilidade, perdas reprodutivas e econômicas. É considerada como uma das principais patologias do aparelho reprodutivo de touros zebuínos, tendo em vista suas características anatômicas e morfológicas. Este estudo descreve um caso de acrobustite diagnosticada em um touro Nelore. No exame clínico, observou-se aumento de volume e tamanho no prepúcio, com consistência firme à palpação com formação de tecido granulomatoso, estenose do óstio prepucial, extremidade do pênis voltada caudalmente e com impossibilidade de exposição do pênis. O tratamento escolhido foi à a postoplastia, com amputação parcial da porção prepucial acometida. Utilizando-se no pós-operatório limpeza da ferida, ducha com água fria, antibioticoterapia a base de penicilina associada com Piroxicam, pomada cicatrizante e Bactrovet Prata. Dessa forma, o procedimento cirúrgico foi executado com êxito obtendo uma rápida recuperação do animal, permitindo com que o mesmo retornasse a vida reprodutiva 60 dias após o procedimento cirúrgico.

Palavras chaves: Bovino, inflamação do prepúcio, reprodução

\section{Postoplasty in Nellore bull affected with acrobustitis: Case report}

Abstract. The objective of this work was to describe a case of acrobustitis, characterized by inflammation of the foreskin tip in bulls, causing infertility, reproductive and economic losses. It is considered as one of the main pathologies of the reproductive system of zebu bulls, given its anatomical and morphological characteristics. This study describes a case of acrobustitis diagnosed in a Nellore bull. In the clinical examination, an increase in volume and size in the foreskin was observed, with firm consistency on palpation with the formation of granulomatous tissue, stenosis of the preputial ostium, the tip of the penis turned tail, and with the impossibility of exposing the penis. Postoperative treatment was chosen, with partial amputation of the affected preputial portion. In the postoperative period cleaning the wound, shower with cold water, antibiotic therapy based on penicillin associated with Piroxicam, healing ointment, and Bactrovet Silver. Thus, the surgical procedure was successfully performed, obtaining a fast recovery of the animal, allowing it to return to reproductive life 60 days after the surgical procedure.

Keywords: Cattle, inflammation of the foreskin, reproduction 


\section{Postioplastia en toro Nellore afectado con acrobustitis: Informe de caso}

Resumen. El objetivo de este trabajo fue describir un caso de acrobustitis, caracterizada
por la inflamación de la punta del prepucio en toros, causando infertilidad, pérdidas
reproductivas y económicas. Es considerada como una de las principales patologías del
sistema reproductivo de los toros cebú, en vista de sus características anatómicas y
morfológicas. Este estudio describe un caso de acrobustitis diagnosticada en un toro
Nellore. En el examen clínico, se observó un aumento en el volumen y el tamaño del
prepucio, con una consistencia firme a la palpación con formación de tejido granulomatoso,
estenosis del ostium prepucial, la punta del pene volteada caudalmente y con imposibilidad
de exponer el pene. Se eligió el tratamiento postioplastia, con amputación parcial de la
porción prepucial afectada. En el postoperatorio, se realizó limpieza de la herida, se
utilizaron duchas de agua fría, antibioticoterapia basada en penicilina asociada con
piroxicam, pomada cicatrizante y Bactrovet Silver. Por lo tanto, el procedimiento
quirúrgico se realizó con éxito, obteniendo una recuperación rápida del animal,
permitiéndole volver a la vida reproductiva 60 días después del procedimiento quirúrgico.

Palabras clave: Ganado, inflamación del prepucio, reproducción

\section{Introdução}

O Brasil apresenta um número efetivo de bovinos acimas 220 milhões de cabeça segundo a Confederação da Agricultura e Pecuária do Brasil (ANUALPEC, 2019), número expressivo e que se encontra em constate crescimento para suprir a demanda de produtos de origem animal. No entanto, certos fatores podem interferir negativamente a eficiência reprodutiva dos reprodutores, como doenças que afetam a genitália externa, causando a inviabilização da cópula, queda na taxa de prenhez e descarte de animais prematuramente (Hafez \& Hafez, 2004).

A genitália externa dos touros pode ser acometida por várias enfermidades, que resultam em queda da produção, incluindo acrobustite, abscessos, hematomas e lacerações prepuciais, os fibropapiloma de glande e parafimose (Rabelo et al., 2015). Dessas afecções a mais expressiva é a acrobustite, caracterizado pelo processo inflamatório na extremidade do prepúcio com um estreitamento do óstio prepucial com fibrose e impedindo a exposição peniana, levando a impotência coeundi (Rabelo et al., 2008; Rabelo \& Silva, 2011).

A acrobustite é uma enfermidade que tem alta prevalência nos rebanhos brasileiros, e representa cerca de $80 \%$ dos diagnósticos das lesões na genitália externa de touros (Rogério Elias Rabelo et al., 2015). Essa alteração pode ser causado por vários fatores, sendo as principais características morfológicas e anatômicas da bainha prepucial, apresentando-se pendulo, bainha longa, prepúcio longo, abertura do óstio prepucial, problemas no musculo retrator caudal do prepúcio e traumas podem predispor ao surgimento da acrobustite (Silva et al., 2015).

O tratamento clínico é eficiente apenas quando diagnóstico é precocemente, porem as lesões tem quadros de evolução rápido e severo (Anderson, 2008), levando em consideração a isso, a maioria dos casos de acrobustite tem indicação cirúrgica para preservar o reprodutor (Hendrickson, 2010). O presente trabalho objetivou mostrar e descrever o procedimento cirúrgico e forma de manejo no tratamento pós-operatório da acrobustite.

\section{Relato de caso}

No dia oito de março de 2019 foi solicitado o atendimento médico veterinário ao um touro Nelore de 5 anos de idade pesando cerca de $700 \mathrm{~kg}$, apresentando queixa de uma lesão prepucial com 5 meses de evolução. $\mathrm{O}$ animal encontrava-se em uma propriedade na zona rural de Bom Jesus-PI. Durante a anamnese o proprietário relatou que o processo inflamatório se desenvolveu rápido, a partir de uma lesão que o animal sofreu na região prepucial devido a abração mecânica de uma pastagem alta, associada com uma bainha prepucial longa de aproximadamente $28 \mathrm{~cm}$. Além disso, o proprietário relatou ter realizado aplicação de alguns medicamentos à base de antibióticos e anti-inflamatório, e tratamento tópicos com unguento e repelentes contra insetos spray de prata para solucionar o processo 
inflamatório de forma clínica. No entanto, o animal não apresentou melhora significativa e evoluindo para um quadro crônico da patologia.

Durante o exame físico, o animal foi contido em um tronco para verificar os parâmetros fisiológicos do animal e realizar o exame específico. O animal apresentava-se com nível de consciência normal, seu escore corporal estava em torno de 2,5 em uma frequência de 1 a 5, pois devido a dor e o incômodo o animal reduziu seu hábito alimentar e sua busca por alimento nos piquetes, temperatura retal $38,2^{\circ} \mathrm{C}$, a frequência respiratória estava $40 \mathrm{mpm}$ e sua frequência cardíaca aferida era $48 \mathrm{bpm}$, ambas se apresentaram normais e sem alterações no seu funcionamento.

$\mathrm{Na}$ realização do exame específico no sistema reprodutor, o animal possuía os dois testículos e escroto hígidos, na avaliação da lâmina externa do prepúcio foi identificado um desenvolvimento de um tecido espeço de $10 \mathrm{~cm}$ a partir da região distal do prepúcio sem comprometimento do pênis do animal, na porção distal um aumento considerável em seu diâmetro e com a extremidade voltada caladamente, apresentava estenose do óstio prepucial externo e impossibilitado de expor o pênis, na palpação uma consistência firme. Levando em consideração a anamnese e os sinais clínicos, foi dado o diagnóstico de acrobustite-fimose e decidido realizar cirurgia de protoplastia para retirada da porção acometida.

O animal foi preparado para o procedimento cirúrgico, passou por um jejum de 12 horas para sólidos e 8 horas hídrico, após o jejum o animal foi conduzido novamente ao tronco onde foi administrado, acepromazina (Acepran ${ }^{\circledR} 1 \%$ ) como medicação Pré-anestésica na dose de $(0,1 \mathrm{mg} / \mathrm{kg}$ ) intramuscular, depois xilazina $\left(\right.$ Anasedan $^{\circledR}$ ) na dose de $0,3 \mathrm{mg} / \mathrm{kg}$.

Em seguida o troco foi aberto lateralmente e conduziu-se o animal para fora, posicionado o touro foi em decúbito lateral direito sobre uma lona, permitindo uma proteção para os membros torácicos e pélvicos, cabeça e região escapular e bem contido com cordas para garantir segurança durante o procedimento, logo após, foi realizado um bloqueio anestésico circular infiltrativo com Cloridrato de Lidocaína a $2 \%\left(\right.$ Anestt $^{\circledR}$ ) na dose de $5 \mathrm{mg} / \mathrm{kg}$ próximo a região de incisão. Posteriormente, foi feita a tricotomia na região prepucial, e a antissepsia foi feito com a lavagem com água e sabão, e solução de Clorexidina. Após a confirmação da analgesia local, o animal devidamente contido e todo instrumental cirúrgico preparado foi dado início a cirurgia, com o objetivo de remover a porção estenótica do prepúcio.

A técnica escolhida foi a de circuncisão, com a remoção da área lesionada e fixação da mucosa prepucial ao óstio do prepúcio de forma modificada. Inicialmente foi feito a demarcação da região a ser incisada, delimitando a área lesionada da parte íntegra, empregando quatro pinças Kocher equidistantes: cranial, laterais direita e esquerdo, e caudal. Após a demarcação foram posicionadas quatro pinças de Allis nos quatro pontos na mucosa que seriam ligados aos quatro pontos na pele já demarcados, logo foram feitas quatro incisões longitudinais de aproximadamente três centímetros de comprimento no ponto médio entre as pinças de Allis, formando quatro "pétalas". Foi feita a circuncisão da pele do prepúcio, de pinça-a-pinça, para exérese da lesão. Em seguida a divulsão romba e isolamento da mucosa do folheto prepucial interno, pinçando os vasos e submetendo-os a ligaduras com fios absorvíveis e evitando hemorragias. A cooptação da mucosa do folheto prepucial interno à pele do óstio prepucial foi realizado por meio de sutura de reparo padrão Donatti captonado, utilizando fio nylon $\mathrm{n}^{\circ} 2.0$, os captons foram feitos de pedaços de equipo no qual ajudam na diminuição da pressão que o fio faz sobre a pele, diminuindo risco de isquemia local, evitando edema na fase inflamatória

Finalizado a cirurgia, foi feito a antissepsia do local com Solução de Clorexidine e realizado a aplicação dos medicamentos. A conduta pós-operatória consistiu na aplicação de do antimicrobiano Megacilin ${ }^{\circledR}$ PLUS PPU (Benzilpenicilina, Sulfato de dihidroestreptomicina associada com Piroxicam) na dosagem de $20.000 \mathrm{UI} / \mathrm{kg} / \mathrm{IM}$ em dose única, repetida após 72 horas, não sendo usado antiinflamatório, pois essa composição já o apresenta. Além disso foi utilizado Pomada cicatrizante ganadol $^{\circledR}$, uma fina camada cobrindo toda a ferida cirúrgica e Bactrovet Prata $\mathrm{AM}^{\circledR}$ por via tópica. Nos três primeiros dias foi indicado ducha de água fria por 10 minutos, e repouso das atividades reprodutivas. Com 15 dias foi feita uma nova avaliação e o animal apresentava-se ativo, com o processo de cicatrização evoluindo bem, o animal conseguia urinar sem exercer pressão ou dor. Com 60 dias o animal foi reavaliado, conseguindo expor o pênis, local da incisão totalmente cicatrizado e com um 
aumento de ganho de peso. Levando em consideração a isso, o reprodutor foi reintegrado ao piquete junto com as matrizes para retornar à atividade reprodutiva.

\section{Discussão}

O presente relato apresenta um touro da raça Nelore que desenvolveu uma patologia na região prepucial diagnosticada como acrobustite ou acrostite. Devido suas características morfológicas e anatómicas os touros zebuínos tem uma certa disposição por desenvolver essas lesões na lâmina interna do prepúcio, sendo que alguns touros que ultrapassam $36 \mathrm{~cm}$ de prepúcio chegam a ser descartados por grande chance de desenvolverem essa enfermidade (Rabelo \& Silva, 2011). A consequência dessas lesões é o surgimento de um processo inflamatório com o crescimento de um tecido granulo e estenose prepucial, denominado como acrobustite-fimose ou somente acrobustite. Além disso, a maioria dos animais que são criados em sistema extensivos estão em contato com pastagens altas, abrasivas ou com algum tipo de degradação, facilitando a ocorrência de traumas nesses animais e acarretando prejuízos econômicos (Fernandes et al., 2015; Peron \& Evangelista, 2004).

A patologia causa muitas perdas econômica, principalmente quando acomete reprodutores de alto valor genético, o animal do presente relato trata-se de um reprodutor, que é criado em sistema extensivo e foi encontrado com grande edema na região prepucial, perda da libido, perda da habilidade de efetuar a copula, e não conseguia expor o pênis, sinais clínicos sugestivo de Acrobustite, no qual é descrito por Rabelo \& Silva (2011) como: dificuldade ou impossibilidade de efetuar a cópula, edema, necrose da mucosa prolapsada, miíases, hemorragia, abscesso, hipertermia local e disúria

O tratamento da acrobustite depende de alguns fatores para que se tenha sucesso na terapêutica, tendo em vista levar em consideração a porção que foi afetada pelo processo inflamatório, o valor zootécnico do animal e as possíveis perdas da eficiência reprodutiva do animal após o tratamento (Rabelo et al., 2006). Nesse contexto, optou-se por realizar a postoplastia, devido o animal apresentar um quadro de evolução muito grave com um tecido granulo e rígido e que seria irreversível apenas com condutas clínicas. No início da patologia Marques et al. (1988) e Anderson (2008) descrevem a higienização da área lesionada com antissépticos não irritantes, ducha de água fria na região prepucial, tratamento clínico e repouso sexual. Com isso, o processo inflamatório regredi, ocorrendo um certo alívio do incómodo que o animal sente, no entanto caso não haja sucesso no tratamento clinico o mesmo já auxilia na cirurgia, reduzindo o estresse e facilitando o procedimento.

No pós-operatório, o animal foi submetido a um tratamento com anti-inflamatórios, antibióticos e medicamentos tópicos, e dentro de 15 dias a cicatrização se efetivou por completo. Papazoglou \& Kazakos (2002) descrevem que após a cirurgia é recomendado a utilização de aventais de algodão que envolve a região prepucial que foi recentemente operada, evitando com que essa área entre em contado direto com o solo e ou seja alvo de algo objeto que cause um novo trauma no local, entretanto, essa conduta não foi seguida, tendo em vista que essa faixa pode servir de local de acúmulo de urina, e o $\mathrm{Ph}$ da urina causar uma irritação na mucosa prepucial do touro. $\mathrm{O}$ animal ficou em um piquete com uma pastagem baixa, sem a presença de outros animais até finalizar a cicatrização. Esse método se mostrou eficaz, tendo em vista que o animal teve uma recuperação eficiente, e sem complicações.

Vale ressaltar que o animal durante o período pós-operatório deve ser afastado das atividades reprodutivas, pois isso eleva as chances de ocorrer uma reincidiva no animal ou tornar um caso crônico para o touro (Rabelo et al., 2012). Além disso, antes de realizar o procedimento, o proprietário deve estar ciente, que futuramente deve substituir o reprodutor, pois sua vida reprodutiva pode ter sido reduzida, tem o risco de surgir novas complicações e que o touro vai passar um período afastado da vida reprodutiva após a cirurgia (Kasari et al., 1997). O animal ficou em repouso por 60 dias, sem atividade sexual e depois retornou as atividades reprodutivas normalmente.

\section{Conclusão}

A técnica cirúrgica empregada para a correção possibilitou resultados satisfatórios, essa manobra cirúrgica é bastante empregada em casos de acrobustite em touros de alto valor genético como ocorreu no presente caso descrito, é importante que algumas medidas devam ser usadas para minimizar a ocorrência de acrobustite em bovinos, tais como: ter cuidado com a escolha dos touros, que mesmo 
sendo zebu, escolher aquele que possui menor bainha prepucial possível, tratar possíveis infecções prepuciais o mais rápido possível.

\section{Referências bibliográficas}

Anderson, D. E. (2008). Surgery of the prepuce and penis. Veterinary Clinics of North America: Food Animal Practice, 24(2), 245-251. DOI: https://doi.org/10.1016/j.cvfa.2008.02.002

ANUALPEC. (2019). Anuário da Pecuária Brasileira (20th ed., Vol. 1). Instituto FNP.

Fernandes, J. P. B., Rabelo, R. E., \& Vulcani, V. A. S. (2015). Epidemiologia de enfermidades acometendo a genitália externa de touros no estado de Goiás. Biológico, 77(sSupl. 2).

Hafez, B., \& Hafez, E. S. E. (2004). Reprodução Animal (Vol. 1, p. 513). Manole: São Paulo, Brasil.

Hendrickson, D. A. (2010). Técnicas cirúrgicas em grandes animais (Vol. 1, p. 238 p.). Guanabara Koogan.

Kasari, T. R., McGrann, J. M., \& Hooper, R. N. (1997). Cost-effectiveness analysis of treatment alternatives for beef bulls with preputial prolapse. Journal of the American Veterinary Medical Association, 211(7), 856-859.

Marques, J. A., Marques, L. C., Canola, J. C., \& Cattelan, J. W. (1988). A acropostite-fimose em tourosuma técnica cirúrgica de tratamento. Ciência e Veterinária, 2, 2-3.

Papazoglou, L. G., \& Kazakos, G. M. (2002). Surgical conditions of the canine penis and prepuce. Compendium, 34(3), 204-218.

Peron, A. J., \& Evangelista, A. R. (2004). Degradação de pastagens em regiões de cerrado. Ciência e Agrotecnologia, 28(3), 655-661. DOI: https://doi.org/10.1590/s1413-70542004000300023

Rabelo, R E, Silva, L. A. F., Viu, M. A. O., Romani, A. F., Alves, C. B., Fernandes, J. J. R., \& Castro, C. F. P. (2006). Acrobustite bovina: Revisão de literatura. Revista CFMV, 37(1), 29-36.

Rabelo, Rogério Elias, Silva, L. A. F., Brito, L. A. B., Moura, M. I., Silva, O. C., Carvalho, V. S., \& Franco, L. G. (2008). Epidemiological aspects of surgical diseases of the genital tract in a population of 12,320 breeding bulls (1982-2007) in the state of Goias, Brazil. 9(3), 705-713.

Rabelo, Rogério Elias, Silva, L. A. F., Vulcani, V. A. S., Sant'Ana, F. J. F., Assis, B. M., \& Rabbers, A. S. (2015). Enfermidades diagnosticadas na genitália externa de touros: estudo retrospectivo (2007-2013). Ciência Animal Brasileira, 16(1), 133-143. DOI: https://doi.org/10.1590/1089$\underline{68916 i 130990}$

Rabelo, Rogério Elias, \& Silva, O. C. (2011). Aspectos morfofuncionais, clínicos e cirúrgicos do pênis, prepúcio e testículos de touros.

Rabelo, Rogério Elias, Vulcani, V. A. S., Cardoso, L. D., Dutra, H. T., Helrgiel, P. A., \& Vicentin, F. R. (2012). Aspectos anatômicos e sua relação com as enfermidades do prepúcio e pênis no touro. Revista Científica Eletrônica de Medicina Veterinária, 9(18).

Silva, L. F. M. C., Araujo, E. A. B., Oliveira, S. N., Dalanezi, F. M., Zahn, F. S., \& Papa, F. O. (2015). Retorno à atividade reprodutiva de touro Angus após fimose traumática com obliteração parcial da luz prepucial: Relato de caso. Enciclopédia Biosfera, Centro Científico Conhecer-Goiânia, 11(21), 1702-2015.

Histórico do artigo:

Recebido: 25 de junho, 2020. Aprovado: 30 de julho, 2020.

Disponível online: 3 de noviembre, 2020.
Licenciamento: Este artigo é publicado na modalidade Acesso Aberto sob a licença Creative Commons Atribuição 4.0 (CC-BY 4.0), a qual permite uso irrestrito, distribuição, reprodução em qualquer meio, desde que o autor e a fonte sejam devidamente creditados. 